\title{
PARN Knockdown in Cell Lines Results in Differential and Cell-Specific Alterations in the Expression of Cancer-Associated mRNAs
}

\author{
Nishith Babu ${ }^{1}$, Dechamma Pandyanda Nanjappa ${ }^{1}$, Sandra Nazareth ${ }^{2}$, Srividya \\ Arjuna $^{1}$, Anirban Chakraborty ${ }^{1 *}$
}

\begin{abstract}
Ribonucleases (RNases) is the collective term used for the group of enzymes that are involved in mRNA degradation. The shortening of the poly (A) tail through deadenylation is the preferred mechanism of degradation of most eukaryotic mRNAs and poly (A)-specific ribonuclease (PARN) is the most important player in deadenylation. Besides its primarily role in mRNA stability, PARN is also involved in several non-conventional functions. It is conceivable that a decreased RNase activity can alter the stability of cancer-associated mRNAs and this alteration may be differential in cells of different origin. Methods: The effects of siRNA-mediated knockdown of PARN on the post-transcriptional expression of 16 oncogenes and 18 tumor suppressor genes in cells derived from different lineages (NCI-H460 and NCI-H522; lung cancer) and (HEK-293; kidney) were investigated. Further, the effects of PARN depletion on proliferation and death of the lung cancer cells were investigated. Results: Quantitative real time PCR analysis revealed an cell-specific alteration in the expression of the target onco and tumor suppressor genes upon PARN depletion, differently, for cells derived from different lineages. The tumor suppressor genes showed a consistent pattern of down regulation upon PARN depletion in all the three cell types tested. In contrast, the expression of oncogenes was not consistent; while some oncogenes showed overexpression in HEK 293 cells, the majority of them were downregulated in the lung cancer cells. Further, PARN depletion did not alter the proliferation of lung cancer cells, which was in contrast to previous reports. Conclusion: The results of this study reveal that PARN deficiency leads to an altered stability of cancer-associated mRNA, distinctly, in cells of different lineages. Despite previous reports suggesting a potential therapeutic role of PARN in cancer, our results suggest that PARN may not be an important biomarker, particularly in lung cancer.
\end{abstract}

Keywords: Ribonuclease- siRNA- mRNA degradation- cancer mRNAs- biomarker- exonuclease

Asian Pac J Cancer Prev, 23 (1), 207-215

\section{Introduction}

Almost all mRNAs contain a poly (A) tail at its 3 'end, a stretch of approximately 25-200 adenosine residues added post-transcriptionally, which becomes crucial for its processing, export into the cytoplasm and translation (Dehlin et al., 2000; Garneau et al., 2007; Lee et al., 2012). In addition, the length of the poly(A) tail also influences the expression of mRNAs at different physiological conditions such as development, differentiation, DNA damage response, apoptosis and cancer (Murphy and Kleiman; 2020 Zhang et al., 2015).

Excess mRNAs undergo degradation and it is the balance between its synthesis and degradation that determines the steady-state level of mRNAs. The removal of poly (A) residues of mRNA is called de-adenylation and it is considered the major pathway in mRNA degradation
(Cevher et al., 2010; Chiba et al., 2004). A number of enzymes, collectively called RNases are involved in mRNA degradation (Guhaniyogi et al., 2001). Among the many RNases, the deadenylases are most crucial for deadenylation by virtue of their high affinity to the poly (A) tail of the mRNA (Dhanraj et al., 2015). Poly(A) specific ribonuclease (PARN) is believed to be the key deadenylase in regulating mRNA turnover rates in mammalian cells (Lee et al., 2012). However, contrasting opinion on PARN not being the major deadenylase in mRNA decay is also available (Son et al., 2018; Yamashita et al., 2005). PARN expresses ubiquitously. As per the Human Protein Atlas database (https://www.proteinatlas. org), PARN mRNA displays low tissue specificity and is expressed in all tissues. PARN protein shows cytoplasmic and nuclear expression in most tissues.

It is now becoming increasingly clear that there is

${ }^{1}$ Division of Molecular Genetics and Cancer, Nitte University Centre for Science Education and Research (NUCSER), NITTE (Deemed to be University), Deralakatte, Mangaluru, Karnataka, India. ${ }^{2}$ Department of Veterinary Medicine, College of Veterinary Medicine, National Pingtung University of Science and Technology, Pingtung, Taiwan.*For Correspondence: anirban@nitte.edu.in 
a link between altered post-transcriptional control of mRNAs and cancer. Attenuated degradation of oncogenic mRNAs or enhanced degradation of tumor suppressor mRNAs can contribute to the initiation of carcinogenesis. For instance, a decrease in the activity of RNases targeting oncogene mRNAs or an increase in the activity of RNases targeting tumor suppressor gene $m R N A s$ can potentially result in cancer. Indeed, several RNases have been implicated as oncoproteins and/or tumor suppressors (Kim and Lee 2009). It has been suggested that the deregulation of cancer related genes could be due to the disturbances in cellular mechanisms such as the mRNA decay pathway (Maragozidis et al., 2015).

Over the years, the role of PARN in cancer has garnered considerable attention. Despite its ubiquitous expression, PARN expression is altered in tumour tissues. In fact, it has been proposed to be a potential target for cancer treatment (Balatsos et al., 2012). A number of studies have revealed that PARN expression is mis regulated in cancer including in solid organ cancers and in hematopoetic malignancies For instance, PARN has been shown to be upegulated in acute lymphoid and myeloid leukemia, small cell lung carcinoma (Maragozidis et al., 2012; Maragozidis et al., 2015). Similarly, data from ONCOMINE revealed that PARN is upregulated in many types of cancers including liver, prostate, cervical, colorectal, and esophageal cancer, whereas it is downregulated in triple negative breast cancer patients and in with invasive ductal carcinoma (Zhang and Yan 2015; Miller and Gomez 2017; Gomez-Cambronero 2018). Previously, we have reported that PARN is downregulated in both non-small cell and small cell lung carcinoma (Babu et al., 2021). Studies on cell lines have shown that knockdown of PARN impacts significantly on the global gene expression by altering the mRNA decay (Lee et al., 2012).

In this study, the aim was to investigate whether loss of PARN alters the expression of cancer-associated mRNAs (oncogenic and tumor suppressor mRNAs) and if this alteration is distinctly different between cells derived from malignant and non-malignant lineage. Lung cancer cells were chosen as cells of malignant lineage because in our previous study we found that PARN is consistently downregulated in non-small cell lung cancer patients (unpublished data). Deficiency of PARN was carried out by PARN-specific siRNA transfection, and the transcript levels of select oncogenes and tumour suppressor genes were measured by quantitative real time PCR. Further, the effects of PARN deficiency on proliferation of lung cancer cells were investigated.

\section{Materials and Methods}

\section{Cell Culture}

Commercially available HEK-293 cells (National Centre for Cell Science, Pune) were cultured in Dulbeco's modified Eagle's medium (DMEM, Sigma) containing 10\% Fetal Bovine Serum (FBS, Panbiotech), NCI-H460 (derived from large cell lung carcinoma) and NCI-H522 cell lines (derived from non-small cell lung adenocarcinoma) (National Centre for Cell Science, Pune) were cultured in Rosewell park memorial institute -1640 medium (RPMI, Sigma) containing 10\% Fetal Bovine Serum (FBS, Panbiotech). The cells were grown at $37^{\circ} \mathrm{C}$ in a humidified chamber containing 5\% CO2.

\section{Transfection of cell lines with PARN specific siRNA}

Transfection was carried out by electroporation using Gene Pulser XcellTM Electroporation system (Biorad, USA). Two sets of PARN specific siRNAs were used (Supplementary Table 1). The control siRNA used was MISSION ${ }^{\circledR}$ siRNA Universal Negative Control (Sigma, USA). $100 \mu \mathrm{M}$ of siRNA was used to transfect $10 \times 10^{6}$ cells; post electroporation the cells were seeded onto $100 \mathrm{~cm}^{2}$ culture dishes containing $10 \mathrm{ml}$ of complete growth medium. 72 hours post transfection, the cultures were treated with $5 \mu \mathrm{g}$ of Actinomycin-D for 90 minutes. Efficiency of silencing of PARN was then analysed at both transcript and protein levels by quantitative real time PCR (qRT PCR) and Western blotting respectively. The siRNA that showed higher transfection efficiency in each cell line was chosen for further assays.

\section{Quantitative Real Time PCR}

Total RNA was extracted from the cell lines using RNeasy Minikit (QAIGEN, Germany). $1 \mu \mathrm{g}$ of total RNA was reverse transcribed to cDNA using High-Capacity cDNA Reverse Transcription Kit (Thermo Fisher Scientific, USA). A concentration of $0.5 \mathrm{ng} / \mu \mathrm{l}$ cDNA was used as a template and quantitative PCR was performed for expression profiling of PARN and selected oncogene and tumor suppressor genes in QuantStudio3 Real Time PCR (Thermo Fisher Scientific, USA) using PowerUpTM SYBRTM Green Master mix (Thermo Fisher Scientific, USA). The expression levels were estimated by the $\Delta \Delta \mathrm{CT}$ method. The cycle threshold was normalized against the B-actin and were expressed as absolute values. A total of 16 oncogenes and 18 tumor suppressor genes were selected for analysis and their details including the primer information are given in supplementary Tables 2 and 3.

\section{Western blotting}

Total cell protein was extracted by using $2 \mathrm{X}$ Laemmeli buffer (4\%SDS, 20\%Glycerol, $120 \mathrm{mM}$ Tris- HCl, pH 6.8) and the concentration was estimated using PierceTM BCA Protein Assay (Thermo Scientific, USA). Ten $\mu \mathrm{g}$ of the cell lysate was resolved using 10\% resolving gel by SDSPAGE; the proteins were blotted onto a PVDF membrane. Primary antibodies that were bound to the proteins were detected using HRP conjugated secondary antibodies using ClarityTM western ECL substrate (BioRad, USA) and the chemiluminescence was detected using Chroma HD9 (UVI Tech, UK).

\section{Cell Proliferation Assay}

MTT Assay was performed using Cell Proliferation Kit I (Roche, Germany) to assess cell proliferation, $72 \mathrm{hrs}$ post electroporation. A total of $2 \times 10^{6}$ cells were seeded in triplicate onto a 96 well plate post electroporation in $100 \mu \mathrm{l}$ of complete growth medium. At $72 \mathrm{hrs}$ post seeding the transfected cells, $10 \mu 1$ of MTT reagent was added to each well, incubated in a humidified $\mathrm{CO}_{2}$ incubator maintained at $37^{\circ} \mathrm{C}$ for $4 \mathrm{hrs}$. Later, $100 \mu \mathrm{l}$ of solubilisation solution 
was added and incubated overnight. Following this, the absorbance was checked at $570 \mathrm{~nm}$. Untreated cells were used as positive control and a blank (well containing only medium) was used as a negative control.

\section{Colony Formation Assay}

To assess the colony formation ability of cells, clonogenic assay was performed. Around 10- 100 cells were seeded onto a 6 well plate using suitable culture medium. $72 \mathrm{hrs}$ post electroporation, the cells were fixed using 4\% PFA for 20 minutes, later washed thrice with $1 \mathrm{X}$ PBS. The fixed cells were then stained using $0.2 \%$ crystal violet for 10 minutes. Excess stain was then washed off and dried, the number of colonies were counted. Later $1 \%$ SDS was added to the wells to solubilize the stain; the absorbance was read at $570 \mathrm{~nm}$.

\section{Cell Apoptotic Assay}

Cell apoptotic assay was performed $72 \mathrm{hrs}$ post electroporation. A total of $2 \times 10^{6}$ cells were seeded onto a 96- well plate after electroporation. After $72 \mathrm{hrs}$ of seeding of cells the cells were harvested by trypsinisation. Later, to $25 \mu \mathrm{l}$ cell suspension $1 \mu \mathrm{l}$ of acridine orange/ ethidium bromide (AO/EB) dual stain was added, and the morphology of apoptotic cells was visualised within 20 minutes using a fluorescent microscope (DM 2500, Leica, Germany).

\section{Data Analysis}

The relative quantification $(2-\mathrm{ddCt})$ value obtained using qPCR were used for statistical calculations. The results of PARN siRNA-transfected cell lines were compared with cells transfected with control siRNA. Cell proliferation assay, colony formation assays were also performed in triplicates and compared alongside with the control cell line. All calculations were performed using Microsoft ${ }^{\circledR}$ Excel $^{\circledR} 2013$ tools. The data obtained were compared using Student's t-test, $\mathrm{p}$ value $<0.05$ was considered significant.

\section{Results}

Reduced levels of PARN $m R N A$ and protein after transfection with PARN-specific siRNA

To analyse the efficacy of the siRNAs against PARN, the transcript and protein levels of PARN post transfection were checked by qRT-PCR and western blot respectively. In HEK-293, down regulation of PARN mRNA was seen for both siRNAs (Figure 1A). However, the down-regulation was significant for PARN siRNA2 compared to PARN siRNA1 and hence siRNA2 was used for further experiments. Similarly, in NCI-H460 cells, both the siRNAs significantly downregulated the transcript level of PARN (Figure 1B). However, siRNA1 was considered for further experiments because it resulted in higher level of PARN downregulation. In case of NCI-H522 cell line, PARN siRNA2 showed statistically significant reduction in PARN mRNA level than PARN siRNA1 (Figure 1C) and was considered for further experiments. The relative expression and the $\mathrm{p}$-values for the above obtained results are mentioned in supplementary table 4.

In order to check the efficiency of siRNA in reducing the protein level of PARN, western blot was carried out using a polyclonal human anti-PARN antibody. Anti $\beta$-actin antibody was used as control. A representative image of western blot PARN in HEK293 cells is shown in Figure 1D. PARN protein level was downregulated in both siRNA-transfected cells compared to the control siRNA transfected cell line (Figure 1Db), in accordance with the transcript level data.

PARN depletion alters the transcript levels of oncogene and tumor suppressor $m R N A$ s

Considering the fact that PARN is an important deadenylase involved in mRNA stability, loss of PARN is expected to alter the profiles of mRNAs in a cell. In order
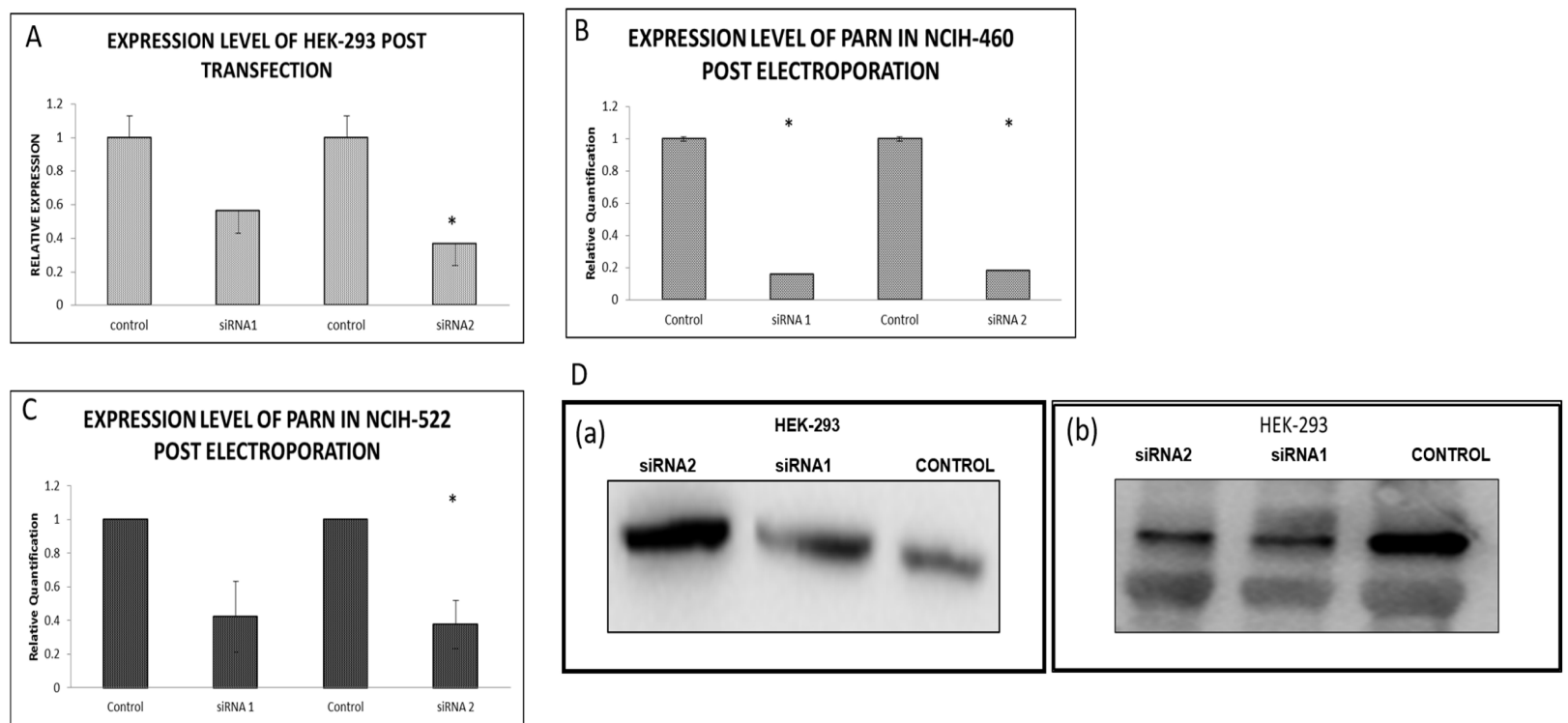

Figure 1. Histogram Illustrating the PARN mRNA Levels in PARN Specific siRNA Transfected Lines of HEK-293 (A), NCI-H460 (B) and NCI-H522 (C) and a representative image of western blot of PARN in HEK293 (Db). $\beta$-actin was used as an internal control (Da) cell lines. 
A

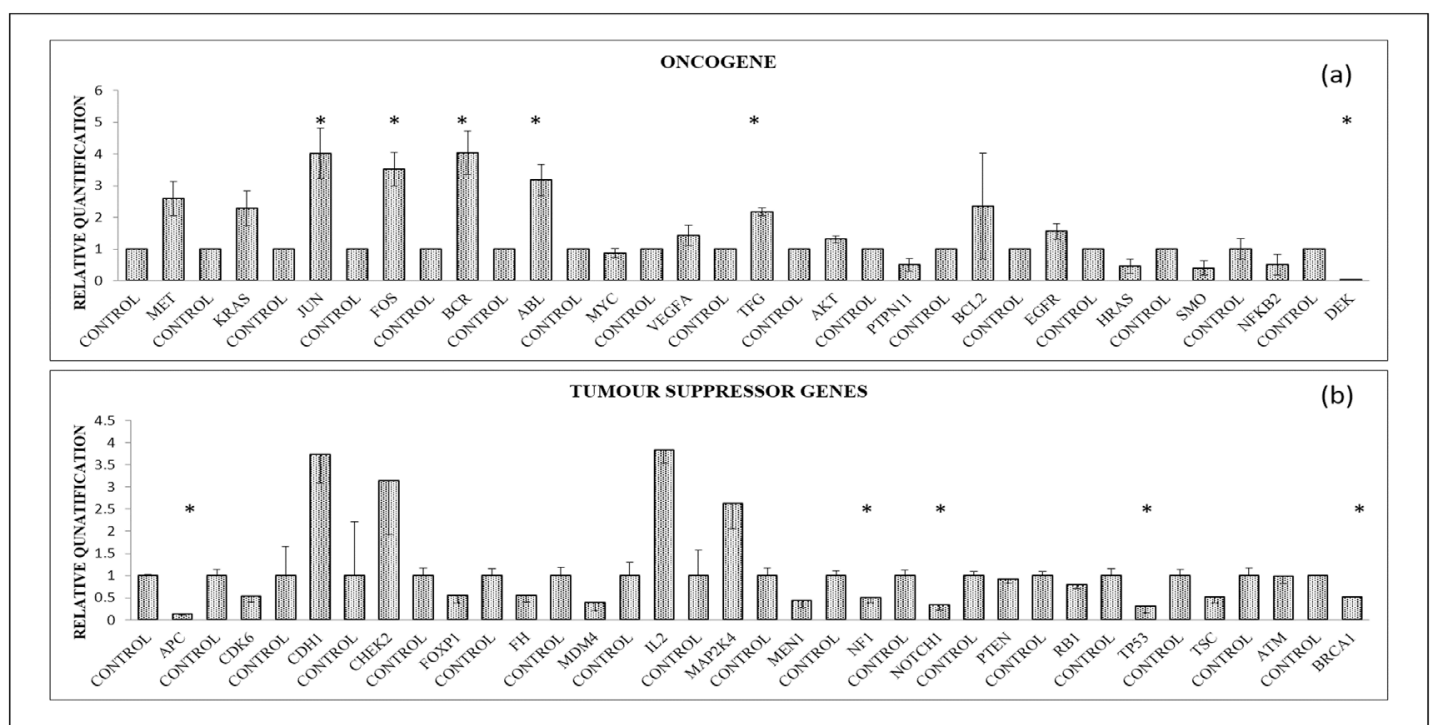

B

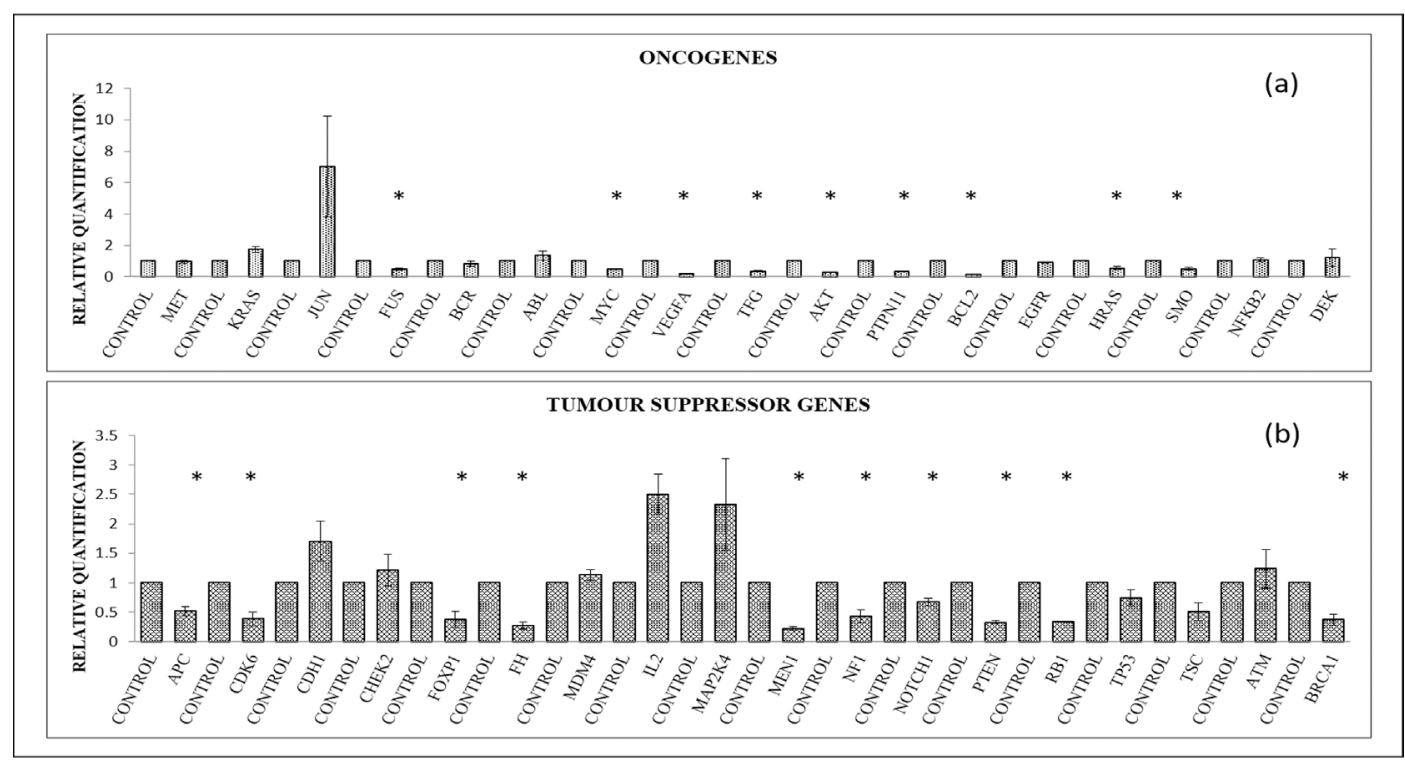

C

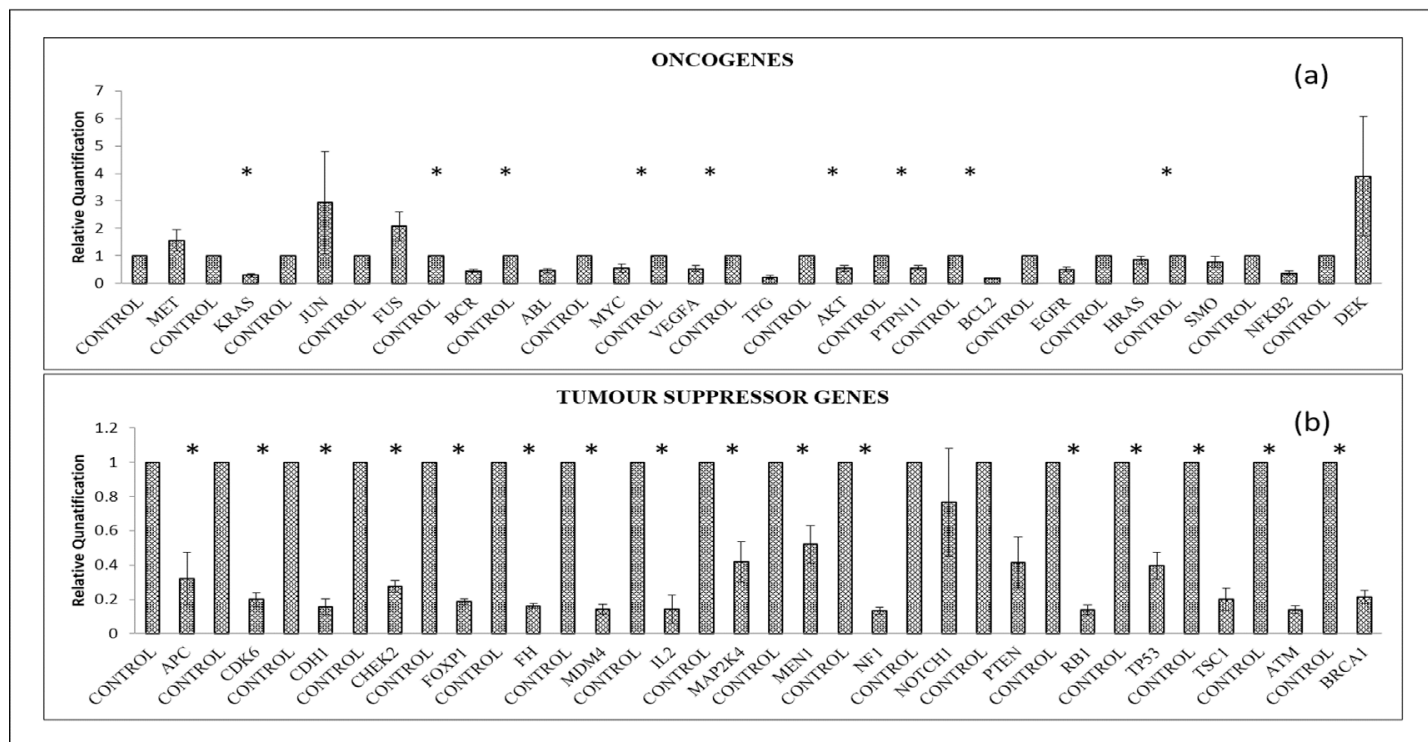

Figure 2. Histogram Showing the Relative Expression of Selected (a) Oncogenes and (b) TSG mRNA levels after transfection with PARN siRNA2 in HEK-293(A), NCI-H460 (B) and NCI-H522 (C), cells normalized to $\beta$-Actin. * indicates $\mathrm{p}<0.05$ 


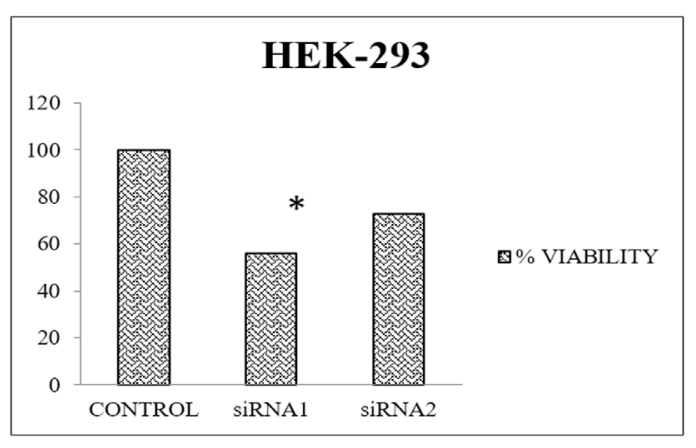

A
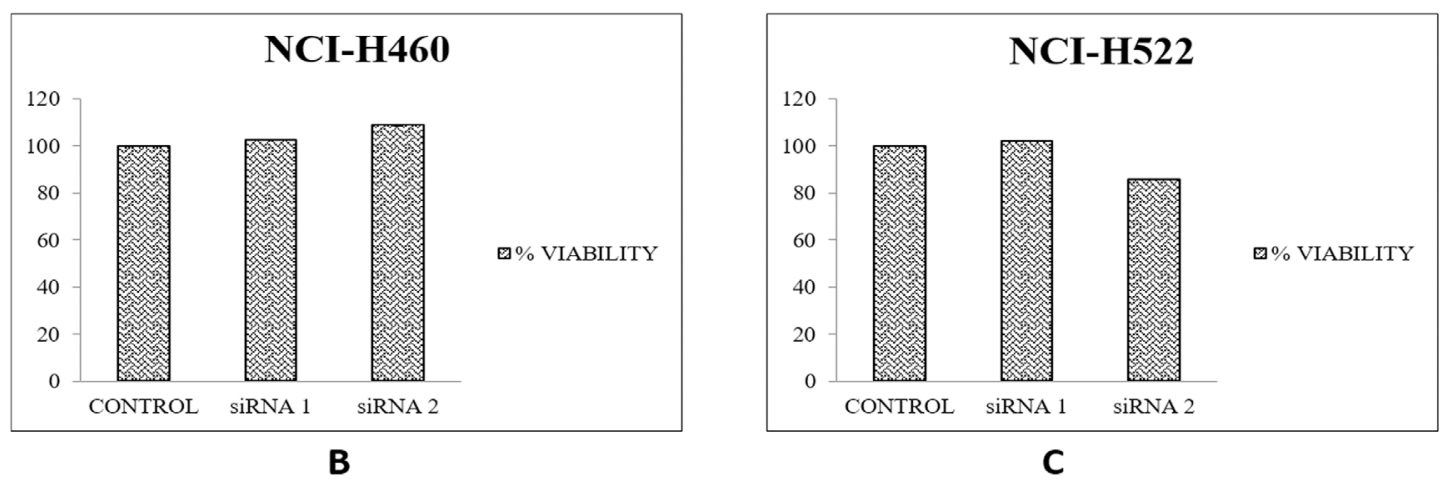

Figure 3. Histogram Showing the Effect of siRNA Transfection on Cell Proliferation as Assessed by MTT Assay for HEK-293 (A), NCI-H460 (B) and NCI-H522 (C) Transfected Cell Lines. * indicates p $<0.05$

to check if the transcript profile of a selected oncogenes and tumor suppressor genes was altered in PARN knocked down cells, the expression of 16 oncogenes and 18 TSGs was analysed by qRT-PCR in PARN siRNA transfected cells and compared to control cells (cells transfected with control siRNA). The relative expressions of various genes were normalized to $\beta$-Actin and a histogram of the gene profile was obtained for each cell line. In order to further check if the profile would change depending on the cell type, both cells from malignant and non-malignant orgin were compared. The results of expression profiling revealed two important observations. One, loss of PARN alters the expression profile of oncogenic and tumor suppressor mRNAs and two, the pattern of alteration varies between cell types. As shown in Figure 2A, the loss of PARN in HEK-293 cells resulted in up regulation of 5 (JUN, FOS, BCR, ABL, and TFG) oncogenes out of 16 analysed. Interestingly, almost all the up regulated genes belonged to the group of transcription factor including AP-1 trasncription factors, nuclear tyrosine kinaes. However, of the 18 tumor suppressor genes analysed, 5 (APC, NF1, NOTCH1, TP53 and BRCA1) were downregulated upon PARN knockdown. Although 4 tumor suppressor genes $(C D H 1, C H E K 2, I L 2$ and $M A P 2 K 4)$ showed up regulation, the difference in the levels compared to control siRNA treated cells was not statistically significant (Figure $2 \mathrm{~A}$ ).

In contrast, the loss of PARN in cells of malignant orgin (NCI-H460 and NCI-H522), led to a downregulation of both oncogenes and tumor suppressor genes and the downregulation was observed for the majority of the genes analysed. As shown in Figure 2B, a total of 9 oncogenes (FOS, MYC, VEGFA, TFG, AKT, PTPN1, BCL2, HRAS and $S M O$ ) showed downregulation in NCI-H460 cells. Interestingly, only 2 of those belonged to the category of AP-1 transcription factors. As for the tumor suppressor genes, 10 (APC, CDK6, FOXP1, FH, MEN1, NF1, PTEN, NOTCH1, RB1, BRACA1) out of 18 genes analysed showed downregulation (Figure $2 \mathrm{~B}$ ) and four of them (APC, NF1, NOTCH1, and BRCA1) showed a pattern similar to that seen in HEK293 cells (Figure 2A).

As expected, the pattern of alteration in NCI-H522 cells was similar to what was seen in NCI-H460 cells as both these cells were derived from malginant lineage. As shown in Figure 2C, a total of 9 oncogenes were downregulated (KRAS, BCR, ABL, VEGFA, TFG, PTPN1, $B C L 2, E G F R$ and NFKB2). Similarly, all the tumor suppressor genes except $N O T C H 1$ and PTEN were also downregulated upon PARN knockdown in NCI-H522 cells (Figure 2C). The relative expression and the p-values for the above obtained results are mentioned in supplementary Table 5 .

Taken together, these results suggest that regardless of the cell type, PARN depletion results in downregulation of tumor suppressor genes whereas the expression of oncogenes alters differently depending on the cell type with overexpression in cells derived from non-malignant lineage and reduced expression in cells derived from malignant lineage.

Depletion of PARN does not affect the proliferation and survival of lung carcinoma cells

The effects of PARN loss of function on the proliferation and survival of lung carcinoma cells were investigated. MTT assay was performed to determine the viability of cells post PARN siRNA transfection. As 


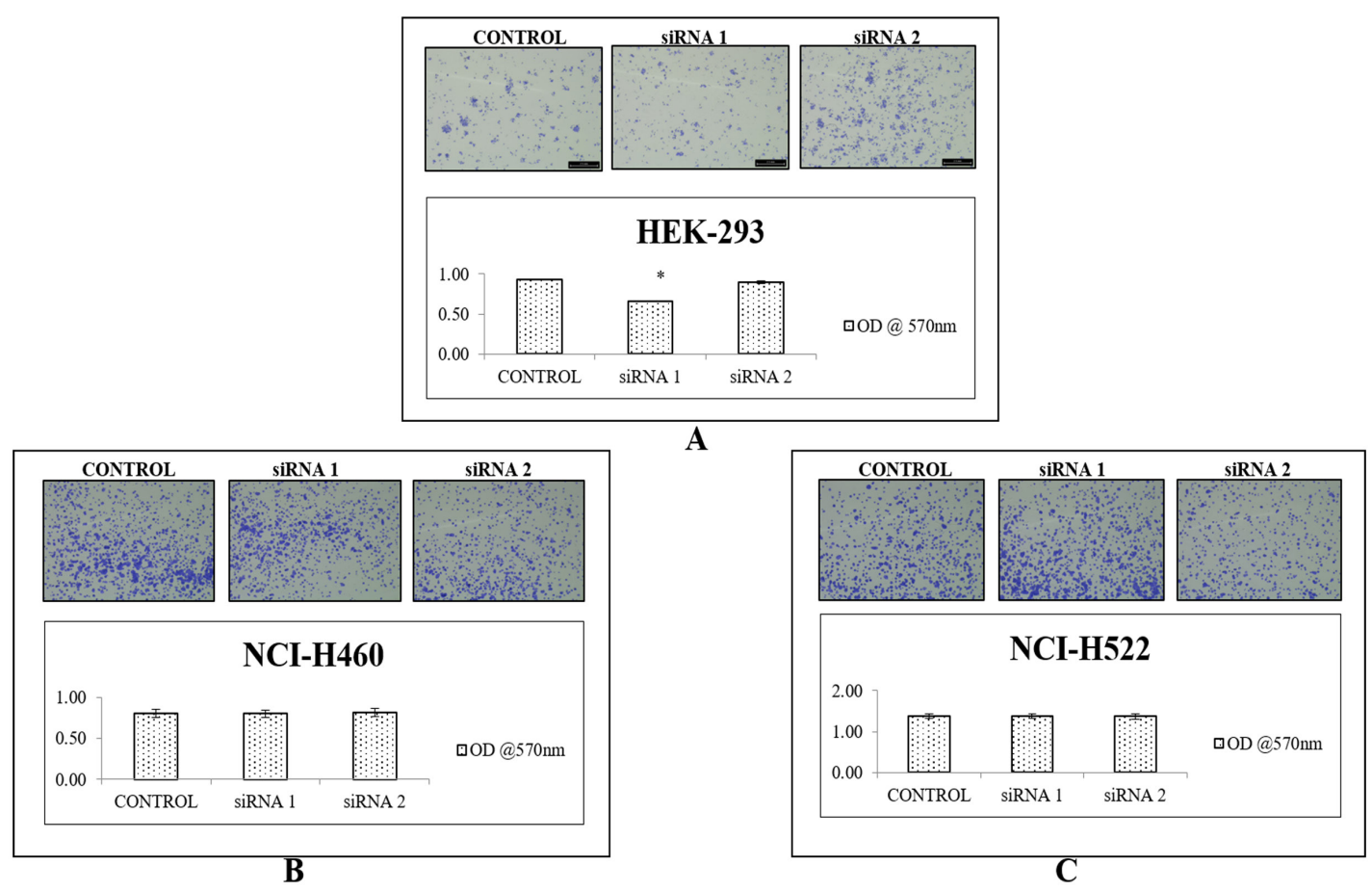

Figure 4. Effect of siRNA Transfection on Colony Formation Assessed by Clonogenic Assay for HEK-293 (A), NCI-H460 (B) and NCI-H522 (C) Transfected Cell Lines. * indicates p $<0.05$.

shown in Figure 3A, there was significant decrease in the cell proliferation rate of HEK-293 cells transfected with PARN siRNA1. However, in NCI-H460 (Figure 3B) and NCI-H522 (Figure 3C) the cell viability remained unaffected compared to control suggesting that depletion of PARN does not inhibit the proliferation of lung carcinoma cells.

Clonogenic assay or colony formation assay is an in vitro assay to check the survival ability of cells. Depletion of PARN with siRNA1 resulted in a statistically significant reduction in colony formation ability of HEK 293 cells, while the ones transfected with siRNA2 did not show any significant difference (Figure 4A). In case of lung carcinoma cells, there was no statistically significant difference in in the absorbance values after PARN silencing (Figures 4B and 4C) compared to controls, suggesting that there was no effect on the colony forming ability of the lung carcinoma cells upon depletion of
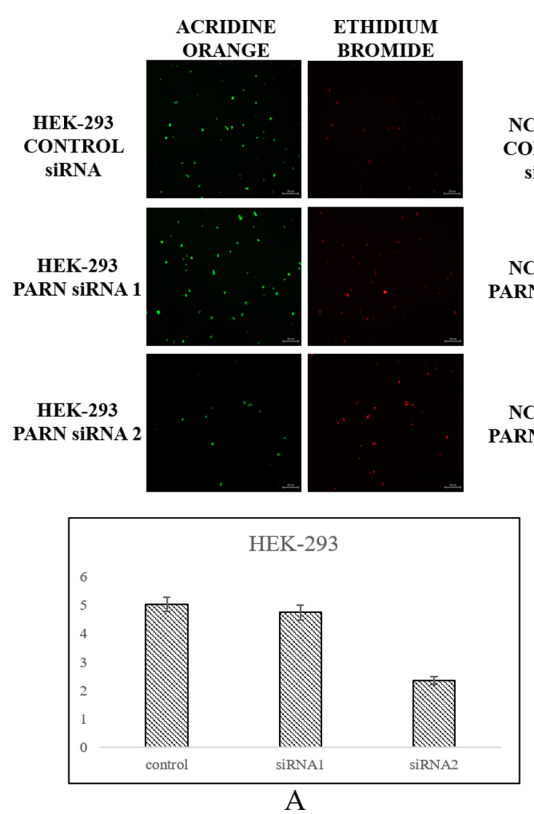
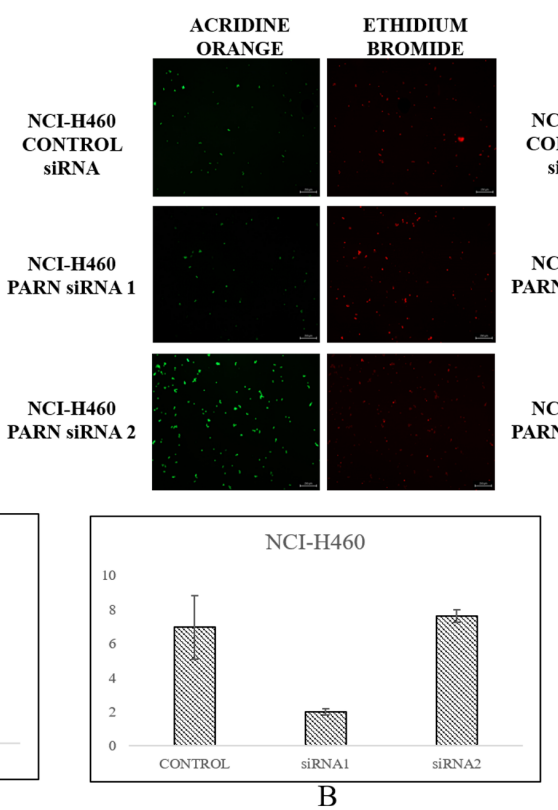
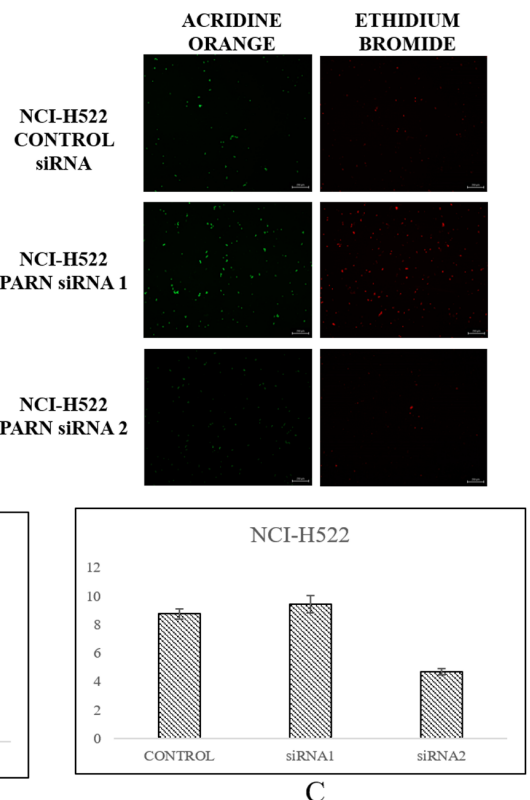

Figure 5. Effect of siRNA Electroporation as Assessed by AO/EB Dual Staining for HEK-293 (A), NCI-H460 (B) and NCI-H522 (C) Transfected Cell Lines. The histogram corresponds to the fluorescence intensity of the captured image which was quantified using ImageJ software. 
PARN

Finally, whether PARN depletion promotes cell death in lung carcinoma cells was analysed by $\mathrm{AO} / \mathrm{EB}$ dual staining. In this assay, the healthy cells take up very less amount of the stain, while apoptotic cells take up acridine orange stain and late apoptotic and necrotic cells take up ethidium bromide stain. On microscopic examination it was observed that there was no significant difference in the number of apoptotic cells both in HEK293 and in lung carcinoma cells treated with PARN siRNA compared to control (Figure $5 \mathrm{~A}-\mathrm{C}$ ), suggesting that the depletion of PARN does not promote cell death in lung carcinoma cells. The relative expression and the $\mathrm{p}$-values for the above obtained results for cell proliferation and clonogenic assay are mentioned in supplementary Table 6.

\section{Discussion}

Deadenylases play crucial role in mRNA regulation and a number of these enzymes have been identified in mammalian cells. Among them, PARN is known to play a crucial role, not only because it is considered to be an important deadenylase but also because of its other roles in cell physiology besides the regulation of mRNA, including the regulation of rRNA, telomerase RNA and other non-coding RNAs (Montellese et al., 2017; Moon et al., 2015; Son et al., 2018). Deregulated PARN expression has been a feature in many cancers (Zhang et al., 2015). Moreover, some human disorders particularly those involving the bone marrow and telomere synthesis, have mutations in PARN (Dhanraj et al., 2015; Stuart et al., 2015; Tummala et al., 2015). Considering its differential expression in cancer cells, PARN has been studied for its role as a biomarker in cancer detection or as a therapeutic target (Balatsos et al., 2012; Maragozidis et al., 2012). It is conceivable that loss of PARN expression can selectively up or down regulate cancer-associated mRNAs, which in turn could trigger tumorigenesis (Aktipis et al., 2013).

In this study, the aim was to investigate if depletion of PARN would alter the expression of cancer-associated mRNAs and if it did, whether the pattern would be different for cells of malignant and non-malignant origin. A total of 16 oncogenes and 18 tumor suppressor genes were selected as cancer-associated mRNAs based on their importance in cancer development and the expression of these target genes was analysed in PARN-deficient cells. The results of this study clearly indicate that PARN deficiency alters the expression of oncogenic and tumor suppressor mRNAs, differently in cells of malignant and non-malignant origin. The expression of tumor suppressor genes was consistently downregulated in PARN-deficient cells whereas the oncogenic mRNAs showed variable expression between cells of malignant and non-malignant origin.

Among the many tumor suppressor genes analysed, the expression of TP53 in the context PARN requires attention. During non-stressed conditions, PARN regulated the expression of TP53 mRNA through de-adenylation of its 3'UTR, thereby maintaining the expression at low level (Devany et al., 2013; Kaeser et al., 2004). Therefore, PARN depletion is expected to result in an upsurge in the expression of TP53 mRNA. However, the results of this study show that depletion of PARN reduces the expression of TP53 mRNA. In HEK293 cells, depletion of PARN resulted in a significant downregulation and the same was observed for NCI-H522 cells (Figure 3A and $3 \mathrm{C}$ ). However, there was no change in TP53 levels upon PARN depletion in NCI-H460 cells. Interestingly, the TP53 gene is wild type in HEK-293 and NCI-H460 cell lines whereas it is mutated in NCI-H522 cells. The fact that PARN depletion did not result in any significant change in the proliferation, survivability or cell death in any of the cell lines perhaps is due to the fact that depletion of PARN reduces the TP53 level instead of the expected increase as per the results of previously published reports. The observations of this study are in direct contrast with the study performed on gastric cancer cells which showed that TP53 mRNA was unaffected by the amount of PARN in the cell (Zhang and Yan, 2015). Nevertheless, the results of this study strongly indicate that PARN is important for maintaining steady state level of mRNA and its downregulation results in aberrant expression of mRNAs.

A number of previous studies have reported that PARN is overexpressed in cancers, including lung carcinoma (Maragozidis et al., 2015, Zhang et al., 2015) and in vitro studies have shown that loss of PARN significantly inhibited the proliferation of gastric cancer cells and promoted cell death (Zhang and Yan 2015). Therefore, the effects of PARN depletion on lung carcinoma cells were investigated. However, in contrast to previous report, downregulation of PARN did not interfere with the cell proliferative rate and colony formation ability of lung carcinoma cells suggesting that PARN may not be a suitable target in cancer, at least in lung adenocarcinoma. Consistent with these results, our previously published data on PARN expression in non-small cell lung cancer revealed a down regulation in the patients (Babu et al., 2021).

In this study, an attempt was made to understand whether PARN deficiency impacts the stability of a group of mRNAs and whether loss of PARN can induce cell death or inhibit the proliferation of lung carcinoma cells. Although, the number of genes analysed were a selected few, it would be interesting to check the entire transcriptome and look at the global expression of all genes. Given the recent observation that PARN is not the primary nuclease in modulating mRNA poly (A) tails (Son et al., 2018), it may become necessary to look at how depletion of PARN affects the stability of other non-coding RNAs including rRNAs and snoRNAs. Although the cancer cells used in this study were carcinoma of the lungs, it is necessary to compare these results with other cancer cell lines to determine if this alteration is a general phenomenon or is specific to certain cancers. The fact that PARN has impact on discrete mRNAs (Lai et al., 2003; Lee et al., 2012; Zhang et al., 2015) suggest further studies that could delineate its role in mRNA stability, particularly in cancer cell lines. Further, the results of western blotting would be interesting to see whether PARN expresses as a single isoform or other phosphorylated forms of this protein in certain cancer conditions. However, the results obtained in this study can form the basis for 
further functional studies on the role of PARN in cellular processes, other than mRNA degradation.

\section{Author Contribution Statement}

NB was involved in performing majority of the laboratory experiments, data analysis and wrote the first draft of the manuscript. DPN was involved in analysis of data and contributed in writing the first draft of the manuscript. SN was partly carried out the laboratory experiments. SA was involved in interpretation of data. AC conceptualised the study, obtained the funds, designed the study protocol, supervised the work, revised the first draft, prepared and edited the final version of the manuscript.

\section{Acknowledgements}

We would like to thank NITTE (Deemed to be University) for providing the research facilities to carry out the above work. The financial support from DST-SERB, Govt. of India, in the form of an Early Career Research Grant to AC (Grant No. ECR/2016/000798) is gratefully acknowledged.

\section{Funding statement}

The financial support for this study was from DST-SERB, Govt. of India, in the form of an Early Career Research Grant to AC (Grant No. ECR/2016/000798).

\section{Scientific Committee Approval}

The research was approved by the Institutional Research and Scientific Committee, Nitte University Centre for Science Education and Research (NUCSER), NITTE (Deemed to be University).

\section{Ethical Approval and consent to participate}

The study did not require any ethical approval as it did not include any animal or human subject.

\section{Statement Conflict of interest}

The authors declare that they have no conflict of interest.

\section{References}

Aktipis CA, Nesse RM (2013). Evolutionary foundations for cancer biology. Evol Appl, 6, 144- 59.

Babu N, Nanjappa DP, Arjuna S, V R, Shetty V, Chakraborty A (2021). Poly(A)- Specific Ribonuclease shows altered expression in solid tumours and hematopoietic malignancies. J Clin Diagnostic Res. (In press).

Balatsos NA, Maragozidis P, Anastasakis D, Stathopoulos C (2012). Modulation of poly(A)- specific ribonuclease (PARN): current knowledge and perspectives. Curr Med Che, 19, 4838-49.

Benjamin D, Moroni C (2007). mRNA stability and cancer: an emerging link?. Expert Opin Biol Ther, 7, 1515-29.

Cevher MA, Zhang X, Fernandez S, et al (2010). Nuclear deadenylation/polyadenylation factors regulate 3' processing in response to DNA damage. $E M B O J, 29,1674-87$.

Dana H, Chalbatani GM, Mahmoodzadeh H, et al (2017). Molecular mechanisms and biological functions of siRNA.
Int J Biomed Sci, 13, 48-57.

Dehlin E, Wormington M, Korner CG, Wahle E (2000). Cap-dependent deadenylation of mRNA. EMBO J, 19, 1079-86.

Devany E, Zhang X, Park JY, Tian B, Kleiman FE (2013). Positive and negative feedback loops in the $\mathrm{p} 53$ and mRNA 3' processing pathways. Proc Natl Acad Sci U S A, 110, 3351-6.

Dhanraj S, Gunja SM, Deveau AP, et al (2015). Bone marrow failure and developmental delay caused by mutations in poly(A)-specific ribonuclease (PARN). J Med Genet, 52, 738-48.

Garneau NL, Wilusz J, Wilusz CJ (2007). The highways and byways of mRNA decay. Nat Rev Mol Cell Biol, 8, 113-26.

Gomez-Cambronero J (2018). Lack of effective translational regulation of PLD expression and exosome biogenesis in triple-negative breast cancer cells. Cancer Metastasis Rev, 37, 491-507.

Guhaniyogi J, Brewer G (2001). Regulation of mRNA stability in mammalian cells. Gene, 265, 11-23.

Kaeser MD, Pebernard S, Iggo RD (2004). Regulation of p53 stability and function in HCT116 colon cancer cells . J Biol Chem, 279, 7598-605.

Kim WC, Lee CH (2009). The role of mammalian ribonucleases (RNases) in cancer. Biochim Biophys Acta, 1796, 99-113.

Lai WS, Kennington EA, Blackshear PJ (2003). Tristetraprolin and its family members can promote the cell-free deadenylation of AU-rich element-containing mRNAs by poly(A) ribonuclease. Mol Cell Biol, 23, 3798-812.

Lee JE, Lee JY, Trembly J, et al (2012). The PARN deadenylase targets a discrete set of mRNAs for decay and regulates cell motility in mouse myoblasts. PLoS Genet, 8, e1002901.

Maragozidis P, Karangeli M, Labrou M, et al (2012). Alterations of deadenylase expression in acute leukemias: evidence for poly(a)-specific ribonuclease as a potential biomarker. Acta Haematol, 128, 39-46.

Maragozidis P, Papanastasi E, Scutelnic D, et al (2015). Poly (A)-specific ribonuclease and Nocturnin in squamous cell lung cancer: prognostic value and impact on gene expression. Mol Cancer Res, 14, 187.

Miller TE, Gomez-Cambronero J (2017). A feedback mechanism between PLD and deadenylase PARN for the shortening of eukaryotic poly(A) mRNA tails that is deregulated in cancer cells. Biol Open, 6, 176-86.

Montellese C, Montel-Lehry N, Henras AK, et al (2017). Poly (A)-specific ribonuclease is a nuclear ribosome biogenesis factor involved in human 18S rRNA maturation. Nucleic Acids Res, 45, 6822-36.

Moon DH, Segal M, Boyraz B, et al (2015). Poly (A)-specific ribonuclease (PARN) mediates 3'-end maturation of the telomerase RNA component. Nat Genet, 47, 1482-8.

Murphy MR, Kleiman FE (2020). Connections between 3' end processing and DNA damage response: Ten years later. Wiley Interdiscip Rev RNA, 11, e1571.

Son A, Park JE, Kim VN (2018). PARN and TOE1 constitute a 3 ' end maturation module for nuclear non-coding RNAs. Cell Rep, 23, 888-98.

Stuart BD, Choi J, Zaidi S, et al (2015). Exome sequencing links mutations in PARN and RTEL1 with familial pulmonary fibrosis and telomere shortening. Nat Genet, 47, 512-7.

Tummala H, Walne A, Collopy L, et al (2015). Poly (A)-specific ribonuclease deficiency impacts telomere biology and causes dyskeratosis congenita. J Clin Investig, 125, 2151-60.

Yamashita A, Chang TC, Yamashita Y, et al (2005). Concerted action of poly (A) nucleases and decapping enzyme in mammalian mRNA turnover. Nat Struct Mol Biol, 12, 1054-63. 
Zhang X, Devany E, Murphy MR, et al (2015). PARN deadenylase is involved in miRNA-dependent degradation of TP53 mRNA in mammalian cells. Nucleic Acids Res, 43, 10925-38.

Zhang LN, Yan YB (2015). Depletion of poly (A)-specific ribonuclease (PARN) inhibits proliferation of human gastric cancer cells by blocking cell cycle progression. BBA Mol Cell Res, 1853, 522-34.

\section{(c) (i) (8)}

This work is licensed under a Creative Commons AttributionNon Commercial 4.0 International License. 